\title{
Viroses du houblon en Alsace
}

\author{
Gérard CLOQUEMIN \& Bernard WALTER (*) \\ avec la collaboration technique de Jean DEFFINIS \& Michel Muller (**) \\ G.R.I.S.P. (l) \\ (*) I.N.R.A., Station de Pathologie végétale, 28, rue de Herrlisheim, F 68021 Colmar \\ (**) COPHOUDAL $\left(^{2}\right)$, 2, rue du Houblon, 67000 Strasbourg
}

RÉSUMÉ

Trois virus ont été décelés par la méthode sérologique ELISA sur houblon en Alsace : le "Hop Mosaic Virus" (HMV), le " Prunus Necrotic Ringspot Virus " (PNRSV), l'" Arabis Mosaic Virus » (AMV) dans respectivement $44,13,8$ et 12,2 p. 100 des cas. Les plantes infectées n'extériorisent pas de symptômes foliaires caractéristiques. L'incidence de ces virus sur les rendements en cônes séchés et en acide $\alpha$ est mise en évidence dans les houblonnières fortement contaminées.

Mots clés additionnels : Hop Mosaic Virus, Prunus Necrotic Ringspot Virus, Arabis Mosaic Virns, sérologie, ELISA, rendement, Humulus lupulus.

Three damaging viruses were detected in hops in Alsace by the ELISA technique. Hop Mosaic Virus (HMV), prunus Necrotic Ringspot Virus (PNRSV) and Arabis Mosaic Virus (AMV) infected 44, 13.8 and 12.2\% of plants respectively. Infected plants did not show typical leaf symptoms. A reduction in the yield of dried cones and -acid was obvious in highly contaminated hop gardens.

Additional key words : Hop Mosaic Virus, prunus Necrotic Ringspot Virus, Arabis Mosaic Virus, serology, ELISA, yield, Humulus lupulus.

\section{INTRODUCTION}

La culture du houblon (Humulus lupulus L.) est traditionnelle en Alsace, dans le Nord et en Bourgogne. Pour la fabrication de la bière, deux des principes actifs du houblon sont pris en considération par les brasseurs : les acides amers (acide $\alpha$ ) et les huiles essentielles. En Alsace, certaines origines de houblon sont réputées pour leur valeur aromatique (huiles essentielles) et sont recherchées par les brasseries françaises et étrangères.

En 15 ans, les surfaces houblonnières ont diminué en Alsace de 50 p. 100 et la teneur en acide $\alpha$ est tombée pour la var. "Northern Brewer " de 11 à 7 p. 100 et pour la var. "Brewers Gold» de 7 à 5 p. 100.

Cette baisse pourrait être due à la présence de viroses. En effet ces maladies ont une action négative sur le rendement et la qualité des récoltes: végétation

( $\left.{ }^{1}\right)$ G.R.I.S.P. : Groupement Régional d'Intérêts Scientifique et Phytosanitaire.

$\left({ }^{2}\right)$ COPHOUDAL : Coopérative des Houblonnières d'Alsace. affaiblie, perte de 30 à 70 p. 100 en poids sur la production de cônes, chute du taux d'acide $\alpha$ (SCHMIDT et al., 1970). Mais il s'agit très fréquemment d'une action conjuguée de plusieurs virus qui sont rarement présents isolément dans la plante.

Le houblon peut héberger de nombreux virus dont les plus répandus appartiennent aux 3 groupes suivants :

- Carlavirus : virus de la mosaïque du houblon ou «Hop Mosaic Virus» (HMV), virus latent du houblon ou « Hop Latent Virus » (HLV), virus latent américain du houblon ou "American Hop Latent Virus " (AHLV) (ADAMS et al., 1983). Ils sont transmis par pucerons (Phorodon humuli Schrank, Myzus persicae Sulzer, Macrosiphum spp.) selon le mode non persistant.

- Ilarvirus: virus des anneaux nécrotiques du prunier ou "Prunus Necrotic Ring Spot Virus" (PNRSV). Plusieurs sérotypes sont décrits: le sérotype "pommier », le sérotype « cerisier » et des sérotypes intermédiaires (BOCK, 1967 ; BARBARA et al., 1978). Il est transmis par le pollen.

- Nepovirus : virus de la mosaïque de l'arabis ou «Arabis Mosaic Virus» (AMV) (Bock, 1966). Il est 
transmis par le nématode Xiphinema diversicaudatum (VALDEZ et al., 1974 ; THRESH \& PITCHER, 1978).

Dans certains pays le houblon fait l'objet d'une sélection sanitaire vis-à-vis des principales viroses. Les techniques de thermothérapie et de culture de méristèmes sont employées (SCHMIDT \& WÖLBLING, 1983).

Des gains de rendement de 3 à 16 p. 100 et des augmentations de 9 à 16,2 p. 100 de la teneur en acide $\alpha$ ont été enregistrés à la suite de la plantation de houblons indemnes de viroses.

Le travail que nous menons sur l'ensemble des houblonnières alsaciennes a 2 objectifs distincts :

- connaître l'état sanitaire des houblons et étudier la relation entre la présence de virus et les diminutions de rendements observées ;

- chercher, au sein des meilleures origines de houblons, des plantes saines en vue d'une multiplication.

Dans cet article sont présentés les résultats de notre prospection concernant 3 virus importants sur houblon (AMV, HMV et PNRSV) et ceux de l'application de la méthode immuno-enzymatique ELISA (THRESH et al., 1977) à leur identification.

\section{MATÉRIEL ET MÉTHODES}

\section{A. Matériel végétal}

Les 2 variétés de houblon les plus cultivées en France sont les variétés anglaises « Northern Brewer » (NB) et «Brewers Gold » (BG), initialement riches en acide $\alpha$. L'Alsace se caractérise par la présence d'une vieille population de houblons aromatiques: le "Strisselspalt» (SP). La sélection massale du SP menée par certains planteurs a été poursuivie par le clonage.

En 1984, 1174 pieds ont été prélevés sur 8 cultivars de houblon, notés et soumis à l'analyse sérologique. Ces prélèvements ont été effectués dans 11 communes, chez 12 planteurs déterminant ainsi 33 zones dont 15 sont plantées en SP.

Pour une partie des échantillons, sur une même liane, nous avons recherché les virus à la fois dans de très jeunes feuilles (sommet) et dans des feuilles plus âgées (milieu de la plante).

\section{B. Transmission mécanique et hétérogreffage}

Nous avons limité notre recherche à 3 virus : AMV, HMV et PNRSV, écartant pour une $1^{\text {re }}$ approche les virus latents : HLV, très répandu, et AHLV, absent des variétés européennes mais détecté sur des variétés américaines introduites en Allemagne (EPPLER \& SANDER, 1984) et en Grande-Bretagne (ADAMS et al., 1983).

Des essais de transmission à des hôtes herbacés sont faits par inoculation mécanique $(1 \mathrm{~g} / 4 \mathrm{ml}$ de tampon phosphate Sörensen pH 7,1). Dans le but de disposer également de plants de houblon infectés par les différents virus, des houblons sains, issus de semis, sont infectés, soit par inoculation mécanique, soit par la technique d'hétérogreffage in vitro (BASS \& VUITTENEZ, 1979). Dans cette dernière méthode, des frag- ments de tige de plantes herbacées contaminées (Chenopodium quinoa, Vinca rosea, Nicotiana clevelandii) sont greffés sur des tiges de jeunes.houblons sains (fig. 1).

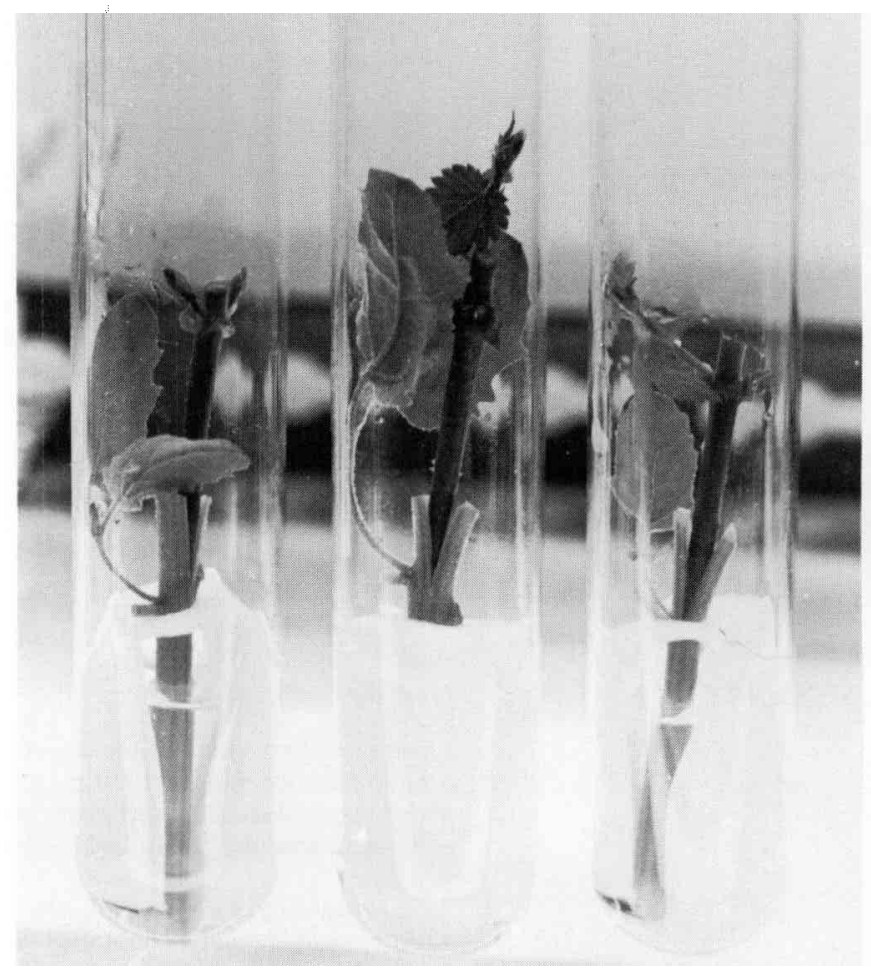

Figure 1

Hétérogreffe in vitro entre Humulus lupulus issu de semis et Chenopodium quinoa infecté par l'AMV.

In vitro heterografting between Humulus lupulus (from seed) and AMV-infected Chenopodium quinoa.

\section{Sérologie}

\section{Les réactifs}

La purification des immunoglobulines s'effectue selon la méthode au rivanol (HARDIE \& VAN REGENMORTEL, 1977). Les IgG purifiées sont conservées à $-20^{\circ} \mathrm{C}$ à une concentration de $1 \mathrm{mg} / \mathrm{ml}\left(\mathrm{E}_{\mathrm{lcm}}\right.$ $0,1$ p. $100=1,4)$.

La phosphatase alcaline est couplée aux IgG en présence de glutaraldéhyde à 0,6 p. 100 (AVRAMEAS, 1969) et les conjugués sont conservés à $4{ }^{\circ} \mathrm{C}$.

\section{Méthode ELISA}

Les tests sont réalisés dans des plaques NUNC $96 \mathrm{~F}$ selon la méthode sandwich directe (CLARK \& ADAMS, 1977). Les antigènes sont préparés en broyant $1 \mathrm{~g}$ de feuille congelée à l'aide d'un broyeur à cylindres hélicoïdaux (presse Pollähne) en présence de $5 \mathrm{ml}$ de tam- 
pon phosphate (PBS) pH 7,4 contenant 1 p. 100 de Tween 20 et 1 p. 100 d'albumine de sang de bœuf. Les extraits sont centrifugés pendant $10 \mathrm{mn}$ à $1500 \mathrm{~g}$. La réaction est mesurée par lecture de la densité optique (D.O.) à $405 \mathrm{~nm}$ avec un lecteur TITERTEK Multiskan. Sont considérés comme contaminés les échantillons dont la D.O. est supérieure à 2 fois la D.O. du témoin constitué par un jus de houblon sain, la D.O. de référence étant ajustée sur une plaque vide.

\section{Mesure de la quantité d'acide $\alpha$}

Les analyses sont effectuées par le laboratoire HOP VALOR de Bischwiller par la méthode E.B.C. (European Brewery Convention) analytica III.

\section{RÉSULTATS}

\section{A. Symptômes sur houblon}

Dans l'ensemble, très rares étaient les plantes présentant des symptômes foliaires. Nous avons observé, lors des prélèvements, quelques symptômes de mosaïque sur feuilles semblables à ceux généralement décrits pour le HMV (fig. 2). Aucune corrélation positive n'a

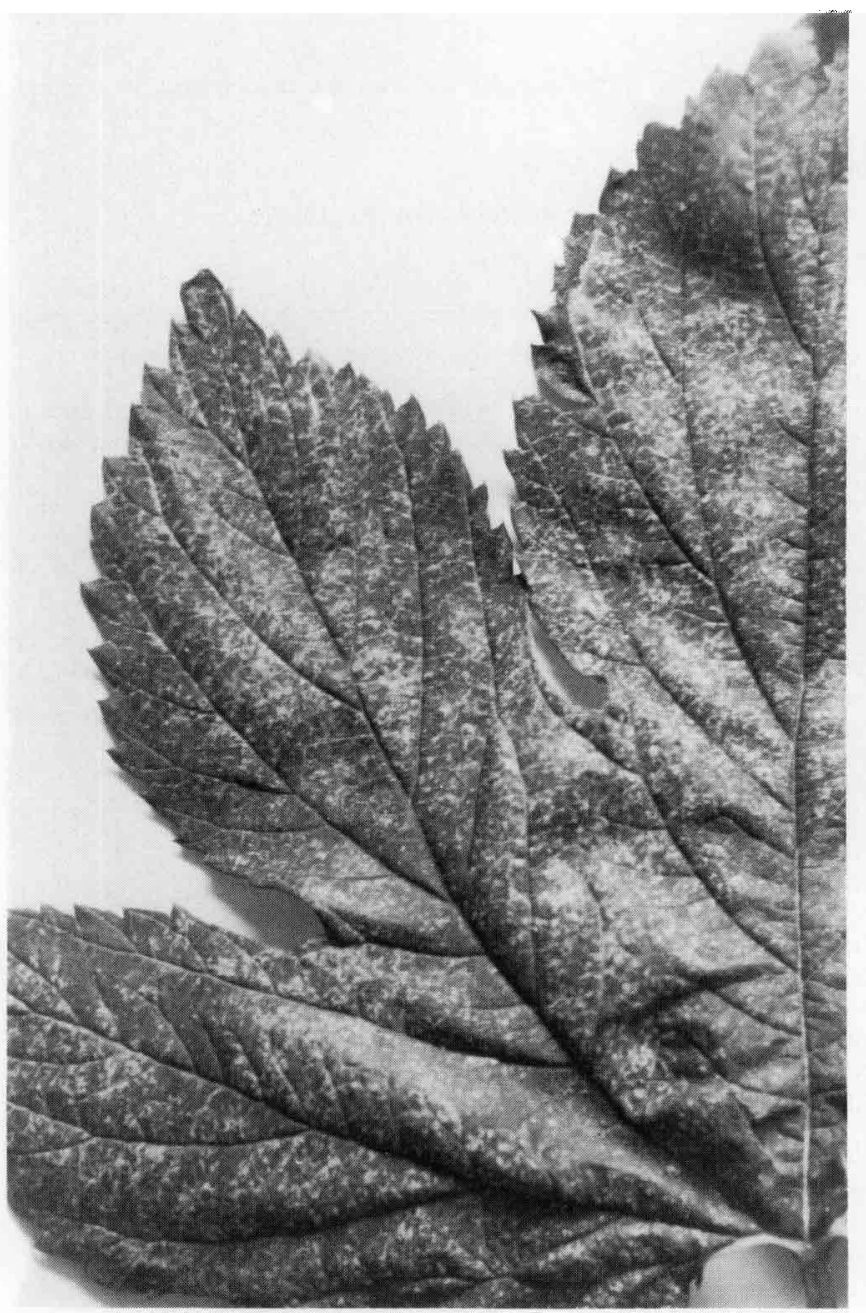

Figure 2

Symptômes de mosaïque sur feuilles de « Brewers Gold", comparables à ceux provoqués par le virus de la mosaïque du houblon (HMV).

Mosaic symptoms on variety 'Brewers Gold' resembling symptoms induced by hop mosaic virus (HMV). pu être établie entre les symptômes et les résultats des analyses par la méthode ELISA.

\section{B. Transmission}

A partir des antigènes conservés sur chlorure de calcium nous avons inoculé une série de plantes herbacées. La réussite de la transmission a été vérifiée par le test ELISA (tabl. 1). Dans aucun cas l'inoculation mécanique sur $H$. lupulus n'a abouti, par contre la technique d'hétérogreffage a permis de transmettre l'AMV à partir de $C$. quinoa, le HMV à partir de $N$. clevelandii. Le PNRSV a pu être transmis à partir de $C$. quinoa mais non à partir de $V$. rosea (tabl. 2).

TABLEAU 1

Inoculations sur hôtes herbacés.

Inoculation of herbaceous test plants.

\begin{tabular}{|c|c|c|c|}
\hline $\begin{array}{l}\text { Anti- } \\
\text { gènes }\end{array}$ & Plante hôte & Symptômes observés & $\begin{array}{c}\text { Test } \\
\text { ELISA }\end{array}$ \\
\hline \multirow[t]{7}{*}{ AMV } & $\begin{array}{l}\text { Chenopodium } \\
\text { amaranticolor }\end{array}$ & $\begin{array}{l}\text { éclaircissement des ner- } \\
\text { vures puis mosaïque, } \\
\text { nécrose apicale }\end{array}$ & + \\
\hline & $\begin{array}{l}\text { Chenopodium quinoa } \\
\text { Cucumis sativus }\end{array}$ & lésions locales chloro- & + \\
\hline & & $\begin{array}{l}\text { tiques sur cotylédons, } \\
\text { mosaïque sur feuilles }\end{array}$ & + \\
\hline & Humulus Iupulus & - & - \\
\hline & Nicotiana tabacum & anneaux chlorotiques & + \\
\hline & Petunia hybrida & marbrure & + \\
\hline & Phaseolus vulgaris & nécrose apicale & + \\
\hline \multirow[t]{6}{*}{ HMV } & Chenopodium album & - & - \\
\hline & Humulus lupulus & - & - \\
\hline & Nicotiana clevelandii & - & + \\
\hline & Plantago major & - & - \\
\hline & Polygonum aviculare & 一 & - \\
\hline & Stellaria media & - & - \\
\hline \multirow[t]{6}{*}{ PNRSV } & Chenopodium quinoa & $\begin{array}{l}\text { plages chlorotiques, feuil- } \\
\text { les déformées }\end{array}$ & + \\
\hline & Cucumis sativus & $\begin{array}{l}\text { taches chlorotiques sur } \\
\text { cotylédons, mosaique sur } \\
\text { feuilles, nécrose apicale }\end{array}$ & + \\
\hline & Cyamopsis tetragonoloba & - & - \\
\hline & Humulus lupulus & - & - \\
\hline & Momordica balsamina & - & - \\
\hline & Vinca rosea & - & + \\
\hline
\end{tabular}

TABLEAU 2

Hétérogreffes Humulus lupulus/hôte herbacé.

Heterografting Humulus lupulus/herbaceous test plants.

\begin{tabular}{|c|c|c|c|c|}
\hline Hôte herbacé & Virus & $\begin{array}{l}\text { Nombre } \\
\text { hétéro- } \\
\text { greffes }\end{array}$ & $\begin{array}{c}\text { Nombre } \\
\text { de plantes } \\
\text { viables }\end{array}$ & $\begin{array}{c}\text { Pourcentage } \\
\text { trans- } \\
\text { mission }\end{array}$ \\
\hline $\begin{array}{l}\text { Chenopodium } \\
\text { quinoa }\end{array}$ & AMV & 28 & 17 & 100 \\
\hline Vinca rosea & PNRSV & 9 & 1 & 0 \\
\hline $\begin{array}{l}\text { Chenopodium } \\
\text { quinoa }\end{array}$ & PNRSV & 9 & 5 & 40 \\
\hline $\begin{array}{l}\text { Nicotiana } \\
\text { clevelandii }\end{array}$ & HMV & 9 & 3 & 33 \\
\hline
\end{tabular}

\section{Application du test ELISA}

\section{Utilisation des réactifs}

Les dilutions d'utilisation des réactifs sont déterminées par des essais préliminaires sur des jus de 
houblon. Nous avons vérifié qu'aucune réaction avec des plantes saines n'était observée et qu'il n'existait pas de réactions avec les antigènes hétérologues.

\section{Niveau de prélèvement}

Les résultats des analyses effectuées sur des feuilles prélevées au sommet d'une liane (environ $2 \mathrm{~m}$ ) et en son milieu concordent. Les écarts de D.O. observés n'ont jamais été de nature à induire le doute dans l'interprétation du test.

\section{Bilan sanitaire}

Comme le montrent les résultats des analyses effectuées par la méthode ELISA (tabl. 3), les 3 virus recherchés sont très répandus et 68 p. 100 des plantes soumises à l'analyse sont infectées par l'un au moins de ces virus. L'AMV et le PNRSV se trouvent souvent associés au HMV omniprésent. La présence simultanée des 3 virus dans une même plante est rare: 3 p. 100 des plantes analysées pour le « $\mathrm{BG}$ » et 0,5 p. 100 pour le «SP ».

La répartition par houblonnière (tabl. 4) des virus recherchés montre que, dans une seule houblonnière $\left(n^{\circ} 11\right)$, l'ensemble des échantillons prélevés s'est révélé exempt des 3 virus; il s'agit d'un clone de "SP » planté en 1981. L'AMV et le PNRSV n'ont pas été décelés dans les échantillons provenant de 2 localisations : Batzendorf (houblonnières 4, 5,6) et Mittelschaeffolsheim (houblonnières 7, 8, 9).

La comparaison des rendements en poids de cônes

TABLEAU 3

Pourcentage de plants des 3 variétés "Brewers Gold", "Northern Brewer », "Strisselspalt " infectés par les virus AMV, HMV et PNRSV. Percentage of AMV, HMV and PNRSV-infected hops of three varieties (Brewers Gold, Northern Brewer, Strisselspalt).

\begin{tabular}{|c|c|c|c|c|c|c|c|c|c|}
\hline Variétés & Sain & $\begin{array}{l}\text { AMV } \\
\text { seul }\end{array}$ & $\begin{array}{l}\text { HMV } \\
\text { seul }\end{array}$ & $\begin{array}{l}\text { PNRSV } \\
\text { seul }\end{array}$ & $\begin{array}{c}\mathrm{AMV}+ \\
\mathrm{HMV}\end{array}$ & $\begin{array}{l}\text { AMV + } \\
\text { PNRSV }\end{array}$ & $\begin{array}{l}\text { HMV + } \\
\text { PNRSV }\end{array}$ & $\begin{array}{l}\text { AMV + HMV } \\
+ \text { PNRSV }\end{array}$ & $\begin{array}{c}\text { Nombre de } \\
\text { plantes }\end{array}$ \\
\hline $\mathrm{BG}$ & 23,5 & 9 & 31,5 & 6 & 15 & 0 & 12 & 3 & 104 \\
\hline NB & 24 & 1,25 & 57 & 1,25 & 3,5 & 0 & 13 & 0 & 322 \\
\hline SP & 64,5 & 8 & 16 & 7 & 1 & 1,5 & 1,5 & 0,5 & 520 \\
\hline
\end{tabular}

TABLEAU 4

Détection des 3 virus AMV, HMV, PNRSV par la méthode ELISA dans les houblonnières en Alsace. Serological detection of $A M V, H M V$ and PNRSV in hop gardens.

\begin{tabular}{|c|c|c|c|c|c|c|c|}
\hline \multirow{2}{*}{ Commune } & \multirow{2}{*}{ Houblonnière } & \multirow{2}{*}{$\begin{array}{c}\text { Année } \\
\text { de } \\
\text { plantation }\end{array}$} & \multirow{2}{*}{ Variétés } & \multirow{2}{*}{$\begin{array}{l}\text { Nombre } \\
\text { de pieds } \\
\text { analysés }\end{array}$} & \multicolumn{3}{|c|}{ Nombre de pieds malades en pourcentage } \\
\hline & & & & & AMV & HMV & PNRSV \\
\hline \multirow[t]{2}{*}{ Furdenheim } & 1 & 1981 & $\mathrm{SP}$ & 50 & - & 22 & 8 \\
\hline & 2 & 1981 & Perle & 50 & - & 4 & 2 \\
\hline Kriegsheim & 3 & 1973 & SP & 50 & 6 & 16 & 28 \\
\hline \multirow[t]{3}{*}{ Batzendorf } & 4 & 1982 & $\mathrm{BG}$ & 22 & - & 100 & - \\
\hline & 5 & $"$ & NB & 12 & - & 75 & - \\
\hline & 6 & $"$ & Perle & 16 & - & 77 & - \\
\hline \multirow[t]{4}{*}{ Mittelschaeffolsheim } & 7 & 1983 & $\mathrm{BG}$ & 10 & - & 100 & - \\
\hline & 8 & $"$ & NB & 20 & - & 95 & - \\
\hline & 9 & " & Perle & 15 & - & 63 & - \\
\hline & 10 & 1982 & $\mathrm{SP}$ & 30 & 7 & 53 & 3 \\
\hline \multirow[t]{2}{*}{ Zeinheim } & 11 & 1981 & SP & 50 & - & - & - \\
\hline & 12 & 1970 & SP & 50 & 6 & 70 & 12 \\
\hline \multirow[t]{3}{*}{ Duntzenheim } & 13 & 1968 & SP & 50 & 38 & 2 & 10 \\
\hline & 14 & 1977 & $\mathrm{SP}$ & 40 & 48 & 20 & 20 \\
\hline & 15 & 1979 & NB & 100 & 9 & 73 & 6 \\
\hline \multirow[t]{3}{*}{ Wittersheim } & 16 & 1968 & $\mathrm{BG}$ & 31 & - & 61 & 90 \\
\hline & 17 & " & NB & 30 & - & 90 & 40 \\
\hline & 18 & 1971 & NB & 30 & - & 20 & 27 \\
\hline \multirow[t]{2}{*}{ Hohatzenheim } & 19 & 1975 & $\mathrm{BG}$ & 50 & 60 & 92 & 20 \\
\hline & 20 & $"$ & NB & 50 & - & 90 & 38 \\
\hline \multirow[t]{8}{*}{ Obernai } & 21 & 1984 & $\mathrm{SP}$ & 30 & - & 13 & 10 \\
\hline & 22 & " & $"$ & $"$ & - & 3 & 3 \\
\hline & 23 & $"$ & $"$ & $"$ & 7 & - & - \\
\hline & 24 & $"$ & $"$ & $"$ & - & 7 & - \\
\hline & 25 & $"$ & $"$ & $"$ & 7 & - & 3 \\
\hline & 26 & $"$ & $"$ & $"$ & 17 & - & - \\
\hline & 27 & $"$ & $\mathrm{BG}$ & 60 & 42 & 32 & 8 \\
\hline & 28 & " & $\mathrm{BG}$ & 30 & - & 30 & - \\
\hline \multirow[t]{4}{*}{ Ingenheim } & 29 & 1984 & NB & 50 & 12 & 44 & 2 \\
\hline & 30 & $" \prime$ & $"$ & 15 & - & 35 & - \\
\hline & 31 & 1972 & $\mathrm{SP}$ & 4 & 75 & - & - \\
\hline & 32 & 1984 & NB & 10 & - & -70 & - \\
\hline Schnersheim & 33 & 1979 & $\mathrm{SP}$ & 16 & - & 75 & 6 \\
\hline
\end{tabular}


TABLEAU 5

Comparaison des rendements (poids de cônes séchés et teneur en acides $\alpha$ ) et des pourcentages de plantes virosées pour la variété "Strisselspalt".

Comparison between cone yields, $\alpha$-acid content and percentage of virus-infected plants for variety 'Strisselspalt'.

\begin{tabular}{|c|c|c|c|c|c|c|}
\hline \multirow{2}{*}{ Houblonnière } & \multirow{2}{*}{$\begin{array}{l}\text { Poids de cônes } \\
\text { séchés (t/ha) }\end{array}$} & \multirow{2}{*}{$\begin{array}{l}\text { Pourcentage } \\
\text { acide } \alpha\end{array}$} & \multirow{2}{*}{ Indice $\mathrm{I}^{*}$} & \multicolumn{3}{|c|}{ Pourcentage de plantes malades } \\
\hline & & & & AMV & HMV & PNRSV \\
\hline 11 & 0,77 & 6,48 & 4,99 & 0 & 0 & 0 \\
\hline 13 et 14 & 0,72 & 5,67 & 4,08 & 42 & 10 & 14 \\
\hline 3 & 0,66 & 4,23 & 2,79 & 6 & 16 & 28 \\
\hline 12 & 0,50 & 4,86 & 2,43 & 6 & 70 & 12 \\
\hline
\end{tabular}

${ }^{*} \mathrm{I}=$ Poids de cônes $\times$ teneur en acides.

séchés par ha et en teneur d'acide $\alpha$ avec les pourcentages de plants virosés dans 4 implantations différentes de la variété «SP » (tabl. 5), confirme que ces rendements sont les plus élevés pour la parcelle dans laquelle aucun des 3 virus recherchés n'a été détecté.

L'incidence calculé $(\mathrm{I}=$ poids de cônes séchés $\times$ teneur en acide $x$ ) est le plus bas pour la houblonnière $\mathrm{n}^{\circ} 12$ dont 70 p. 100 des plantes analysées sont infectées par le HMV (tabl. 4) et 78 p. 100 par l'un au moins des 3 virus.

\section{DISCUSSION ET CONCLUSION}

La présence des virus AMV, HMV et PNRSV dans les houblons en Alsace ainsi que les relations établies entre leur présence et les rendements de la récolte confirment leur incidence sur les diminutions quantitatives et qualitatives de la production houblonnière.

L'évaluation précise de l'impact des virus au champ est difficile à chiffrer puisqu'il existe de nombreux facteurs pouvant influer sur les composantes des rendements : accidents climatiques, âge de la houblonnière, nature du sol, méthodes culturales.

Dans nos prélèvements, 68 p. 100 des plantes sont infectées par au moins un des virus. Le HMV infecte 44 p. 100 des plantes testées ( NB »: 73,5, «BG»: 61,5 et «SP »: 19 p. 100) alors que les chiffres publiés dans d'autres pays sont : Grande-Bretagne, 90 p. 100 (ADAMS et al., 1983), République Fédérale Allemande, 82 p. 100 (ANONYME, 1981) et Portugal, 80 p. 100 (DE VARENNES \& DE SEQUEIRA, 1981).

Le houblon est une plante susceptible d'héberger de nombreux virus, souvent sans symptôme foliaire, ce qui rend nécessaire l'utilisation de méthodes de détection telles que les méthodes sérologiques. Les sérums anglais que nous avons utilisés concernent 3 des plus importants virus décrits jusqu'à présent sur houblon (AMV, HMV, PNRSV). Nous n'avons pas recherché : le Hop Latent Virus (HLV), très répandu mais sans incidence notoire sur les rendements et qui est sérologiquement apparenté au HMV (ADAMS et al., 1983) ; le Cucumber Mosaic Virus (SCHMIDT \& KARL, 1968), le Tobacco Ringspot Virus (SCHMIDT, 1967), rares sur houblon, ni le viroïde du Hop Stunt répandu au Japon (SASAKI \& SHIKATA, 1977).

Certains virus présentent plusieurs sérotypes différents dont la distribution peut varier selon les régions.
Plusieurs souches sérologiquement distinctes de PNRSV ont été décrites (BOCK, 1967 ; BARBARA et al., 1978 ; CASPER, 1983). Les plus répandus sur houblon sont les sérotypes "pommier " (ApMV) et des sérotypes intermédiaires entre l'ApMV et le PNRSV (= sérotype « cerisier »), le sérotype « cerisier » étant relié, mais faiblement, à l'ApMV. Dans 10 p. 100 de nos prélèvements, les souches «pommier 》 et « cerisier » ont été recherchées. Leur présence souvent simultanée nous ont conduits à n'utiliser que les réactifs du sérotype « cerisier ». Une étude plus approfondie des différents sérotypes, et de leur répartition, est en cours.

Dans la perspective d'une lutte ou d'une prophylaxie, la caractérisation des virus, par les gammes d'hôtes et leurs réactions sérologiques, est une étape préliminaire nécessaire. La connaissance des vecteurs et de la répartition de la maladie permettent, dans un second temps, d'échafauder des stratégies de lutte. En ce qui nous concerne, le HMV et ses vecteurs aphides, présents dans les houblonnières, font l'objet d'une protection phytosanitaire justifiée par les déprédations directes des pucerons. Pour l'AMV on ne connaît pas encore la nature et l'importance des populations de nématodes vecteurs en Alsace. Cette information nous permettra de juger le risque de dissémination et de justifier une désinfection des sols dans les houblonnières où l'on souhaite planter du matériel indemne de viroses (MC NAMARA \& PITCHER, 1974).

Pour le PNRSV, le rôle des nématodes n'est pas clairement établi (FULTON, 1970) ; on sait par ailleurs que ce virus peut être transmis par le pollen chez des Prunus (MANDAHAR \& GILL, 1984).

Pour son arôme, la variété traditionnelle alsacienne, " $\mathrm{SP}$ », bénéficie d'un regain d'intérêt de la part des brasseurs. Face à ce marché porteur et dans le but de reconvertir de vieilles houblonnières, l'emploi de matériel indemne de viroses est souhaitable. La recontamination de houblons sains par le PNRSV et par le HMV a été évaluée, 8 années après plantation, à 0,1 et 16,4 p. 100 respectivement pour la variété «Hüller Bitterer " (ANONYME, 1984).

L'obtention de plants sans virus par différentes techniques, est en cours d'étude au GRISP en collaboration étroite avec l'INRA à Colmar.

Reçu le 29 juillet 1985.

Accepté le 28 avril 1986. 


\section{REMERCIEMENTS}

Ce travail a bénéficié du soutien financier de l'Association Nationale du Développement Agricole, dans le cadre du programme houblon de l'Association Générale des Producteurs de Houblon de France.
Nous remercions le Dr. ADAms (East Malling) pour la fourniture des antisérums, ainsi que les agents de la COPHOUDAL, du Service Régional de la Production des Végétaux Alsace et de la Station de Pathologie végétale de l'I.N.R.A. Colmar pour leur collaboration.

\section{RÉFÉRENCES BIBLIOGRAPHIQUES}

Adams A. N., Barbara D. J., Clark M. F., 1983. The occurrence and some properties of three carlaviruses infecting hop plants (Humulus lupulus) in England. Acta Hortic., 130, 243-248.

Anonyme, 1981. Bayerrische Landesanstalt für Bodenkultur $u$ Pflanzenbau - Sonder-nummer, 2 März 82, Jahresberich 1981, p. 57.

Anonyme, 1984. Hopfen - Versuchsergebnisse, Produktionshinweise. Bayerische Landesanstalt für Bodenkultur und Pflanzenbau, Hopfenforschung und Hopfenberatung. Wolnzach/Hüll. Berichts jahr'83, p. 21.

Avrameas S., 1969. Coupling of enzymes to proteins with glutaraldehyde. Use of the conjugates for the detection of antigens and antibodies. Immunochemistry, 6, 43-52.

Barbara D. J., Clark M. F., Thresh J. M., Casper R., 1978. Rapid detection and serotyping of Prunus necrotic ringspot virus in perennial crops by enzyme-linked immunosorbent assay. Ann. Appl. Biol., 90, 395-399.

Bass P., Vuittenez A., 1979. Méthode d'inoculation virale par " hétérogreffage » entre plantes hôtes herbacées et ligneuses en culture aseptique in vitro. Application à la transmission des souches définies de népovirus de Chenopodium quinoa à la vigne Vitis spp. Ann. Phytopathol., 11, 565-567.

Bock K. R., 1966. Arabis mosaic virus and Prunus necrotic ringspot viruses in hop (Humulus lupulus L.). Ann. Appl. Biol., 57, 131-140.

Bock K. R., 1967. Strains of Prunus necrotic ringspot virus in hop (Humulus lupulus L.). Ann. Appl. Biol., 59, 437-446.

Casper R., 1983. ELISA detection of ilarviruses in fruit trees by antisera having strong heterologous reactions. Acta Hortic., 130, 143.

Clark M. F., Adams A. N., 1977. Characteristics of the microplate method of enzyme-linked immunosorbent assay for the detection of plant viruses. J. Gen. Virol., 34, 475-483.

Eppler A., Sander E., 1984. A survey of German hops for the presence of American hop latent virus (AHLV). Phytopathol. Z., 109, 183-187.

Fulton R. W., 1970. Prunus necrotic ringspot virus. $C M I / A A B$ Descriptions of Plant Viruses, $\mathrm{n}^{\circ} 5$.
Hardie G., Van Regenmortel M. H. V., 1977. Isolation of specific antibody under conditions of low ionic strength. J. Immunol. Methods, 15, 305-314

Mandahar C. L., Gill P. S., 1984. The epidemiological role of pollen transmission of viruses. Z. Pflanzenkr. Pflanzensch., 91, 246-249.

McNamara D. G., Pitcher R. S., 1974. The control of arabis mosaic virus in hops by soil fumigation and fallowing. Agro Ecosystems, 1, 123-129.

Sasaki M., Shikata E., 1977. On some properties of hop stunt disease agent, a viroid. Proc. Jap. Acad., 53 B, 109-112.

Schmidt H. E., 1967. New results of hop virus research. Proc. 6th Conf. Czech. Plant Virol., Olomouc, 47-53.

Schmidt H. E., Borde K., Heinrich R., 1970. Untersuchungen zur Frage der wirtschaftlichen Bedeutung von Virosen und virusähnlichen Erscheinungen des Hopfens (Humulus lupulus L.) im Gebiet der DDR. Nachrichtenbl. Pflanzensch., 4, 78-85.

Schmidt H. E., Karl E., 1968. Untersuchungen über eine Scheckung am Japanischen Hopfen (Humulus scandens (Lour.) Merr.). Phytopathol. Z., 62, 272-278.

Schmidt H. E., Wölbing H., 1983. Stand und Perspektive der Produktion von virusfreiem Hopfen. Nachrichtenb. Pflanzensch., 37, $1-4$.

Thresh J. M., Adams A. N., Barbara D. J., Clark M. F., 1977. The detection of three viruses of hop (Humulus lupulus) by enzymelinked immunosorbent assay (ELISA). Ann. Appl. Biol., 87, 57-65.

Thresh J. M., Pitcher R. S., 1978. The spread of nettlehead and related virus diseases of hop, 291-298. In Scott P. R. \& Bainbridge A. : "Plant disease epidemiology". Blackwell Sci. Publ.

Valdez R. B., McNamara D. G., Ormerod P. J., Pitcher R. S., Thresh J. M., 1974. Transmission of the hop strain of arabis mosaic virus by Xiphinema diversicaudatum. Ann. Appl. Biol., 76, 113-122.

De Varennes A., de Sequeira O. A., 1981. A tecnica ELISA ("enzyme-linked immunosorbent assay") na detecçao de virus em plantas. Sua applicaçao na cultura da videira (Vitis vinifera L.) e do lupulo (Humulus lupulus L.). Rep. Congr. Port. Fitiatr. Fitofarm., 305-311. 